\title{
FREE SURFACE FLOW OVER AN OBSTACLE. THEORETICAL STUDY OF THE FLUVIAL CASE
}

\author{
D. BOUKARI, R. DJOUADI, AND D. TENIOU
}

Received 4 August 2001

The two-dimensional stationary flow of a fluid over an obstacle lying on the bottom of a stream is discussed. We take into account the gravity and we neglect the effects of the surface tension. An existence theory for the solution of this problem is established by the implicit function theorem, for small obstacles and Froude numbers in an interval included in ]0, 1[.

\section{Introduction}

This paper considers the problem of determining the free surface flow of an ideal fluid down an infinitely long channel of uniform width. We suppose that the bottom of the channel is perturbed by an obstacle represented by a regular function with a compact support. Free surface flows of an ideal fluid are nonlinear problems; the nonlinearity is essentially due to the dynamic condition written at the free surface.

The practical applications of this type of flow arise both in the hydraulic engineering of fast flow in a channel or a river. The numerical study of this problem has been treated by various authors. Bouhadef [3] has given a numerical study of the problem in the fluvial and torrential case. King and Bloor [6] have given a generalization of the Schwarz-Christoffel transformation to formulate the problem of free streamline jet flow over curved wall as a pair of coupled equations for the tangential angles onto the free surface and the wall shape. Linearized solutions and nonlinear numerical solutions are presented for a variety of wall shapes. But no theoretical result has been given. However several authors have given theoretical results of this problem in the linear case. Abergel and Bona [1] have considered a steady, two-dimensional of an incompressible, Newtonian fluid flowing under gravity down an inclined channel; they have established an 


\section{Free surface fluvial flow}

existence theory for steady, highly viscous flow. In this paper, we want to establish a theoretical result of existence and unicity of a solution which decrease exponentially at infinity. The plan of this paper is as follows.

In Section 2, we formulate the governing equations of the problem in dimensionless form. In Section 3, we introduce the stream function in these equations. In Section 4, the resolution of the dynamic equation written at the free surface boils down to a fixed point problem and here we apply the implicit function theorem. We achieve this work by a conclusion given in Section 5 .

\section{The governing equations}

We consider a steady two-dimensional flow of an ideal fluid in a channel in which an obstacle described by the equation $y=b(x)$ has been placed. We denote by $\Omega_{b}^{\gamma}$ the domain occupied by the fluid, where $b$ is the equation of the obstacle and $\gamma$ is the perturbation of the free surface. We put

$$
\Omega_{b}^{\gamma}=\left\{(x, y) \in \mathbb{R}^{2} \mid-\infty<x<+\infty, b(x)<y<y_{0}+\gamma(x)\right\},
$$

$(x, y)$ is a coordinate system in which $x$ and $y$, respectively, the horizontal and positive vertical directions, see Figure 2.1.

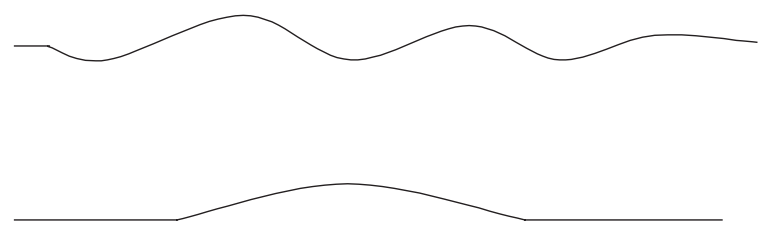

Figure 2.1

The function $b(x)$ verifies $0 \leq b(x)<y_{0}$ and is regular, with a compact support. The problem is formulated as follows: given a bottom configuration $b$, find a function $\gamma: \mathbb{R} \rightarrow \mathbb{R}$ (free boundary) and a vector field $\vec{u}$ (velocity of the fluid) such that:

Governing equations in $\Omega_{b}^{\gamma}$

$$
\begin{aligned}
\operatorname{div} \vec{u}=0 & \text { in } \Omega_{b}^{\gamma}, \\
\operatorname{curl} \vec{u}=0 & \text { in } \Omega_{b}^{\gamma} .
\end{aligned}
$$

Equation (2.2) expresses the incompressibility of the fluid, (2.3) is given by the irrotationality of the flow. 
Boundary conditions

$$
\begin{array}{ll}
\vec{u} \cdot \vec{v}=0 & \text { for } y=b(x), \\
\vec{u} \cdot \vec{v}=0 & \text { for } y=y_{0}+\gamma(x),
\end{array}
$$

where $\vec{v}$ is the exterior normal to the boundary of $\Omega_{b}^{\gamma}$. Equations (2.4) and (2.5) describe the impermeability of the flow at the boundary of the domain $\Omega_{b}^{\gamma}$.

Conditions at infinity. We suppose that the flow is asymptotically uniform and horizontal far upstream and downstream of the obstacle. We then write

$$
\lim _{|x| \rightarrow \infty} \vec{u}(x, y)=\left(u_{0}, 0\right)
$$

hence

$$
\lim _{|x| \rightarrow \infty} \gamma(x)=0
$$

Condition across the free surface. The dynamic condition of continuity of the pressure across the free surface is given by the Bernoulli equation

$$
\frac{\rho}{2}|\vec{u}|^{2}+\rho g y=c
$$

where $\rho$ is the density of the fluid, $g$ is the downward acceleration due to the gravity, and $c$ is a constant.

Dimensionless equations. Dimensionless variables are defined by referring all lengths to the quantity $y_{0}$, and all velocities to $u_{0}$. We put

$$
\vec{u}=u_{0} \vec{u}^{*}, \quad x=y_{0} x^{*}, \quad y=y_{0} y^{*} .
$$

Systems (2.2), (2.3), (2.4), and (2.5) become

$$
\begin{aligned}
\operatorname{div} u^{*}=0, & \text { in } \Omega_{b^{*}}^{\gamma^{*}}, \\
\operatorname{curl} u^{*}=0 & \text { in } \Omega_{b^{*}}^{\gamma^{*},} \\
u^{*} \cdot \vec{v}=0 & \text { for } y^{*}=b^{*}\left(x^{*}\right), \\
u^{*} \cdot \vec{v}=0 & \text { for } y^{*}=1+\gamma^{*}\left(x^{*}\right),
\end{aligned}
$$

where

$$
\begin{gathered}
\Omega_{b^{*}}^{\gamma^{*}}=\left\{\left(x^{*}, y^{*}\right) \in \mathbb{R}^{2} \mid-\infty<x^{*}<+\infty, b^{*}\left(x^{*}\right)<y^{*}<1+\gamma^{*}\left(x^{*}\right)\right\}, \\
b^{*}\left(x^{*}\right)=\frac{1}{y_{0}} b\left(y_{0} x^{*}\right), \quad \gamma^{*}\left(x^{*}\right)=\frac{1}{y_{0}} \gamma\left(y_{0} y^{*}\right) .
\end{gathered}
$$

The conditions at infinity become

$$
\lim _{|x| \rightarrow \infty} \vec{u}^{*}\left(x^{*}, y^{*}\right)=(1,0) .
$$


416 Free surface fluvial flow

The Bernoulli equation takes the form

$$
\frac{F^{2}}{2}\left|\vec{u}^{*}\right|^{2}+1+\gamma^{*}\left(x^{*}\right)=c,
$$

where $F=u_{0} / \sqrt{g y_{0}}$ is the Froude number of the flow.

\section{Formulation of the problem in stream function}

In what follows, we write all the variables without the symbol $*$.

The irrotationality and the incompressibility of the fluid lead us to define a harmonic stream function $\Psi$ such that

$$
\vec{u}=\left(\begin{array}{c}
\frac{\partial \Psi}{\partial y} \\
-\frac{\partial \Psi}{\partial x}
\end{array}\right)
$$

Equation (2.4) will be written as

$$
\left(\begin{array}{c}
\frac{\partial \Psi}{\partial y} \\
-\frac{\partial \Psi}{\partial x}
\end{array}\right) \cdot\left(\begin{array}{c}
b^{\prime}(x) \\
-1
\end{array}\right)=0
$$

and becomes

$$
b^{\prime}(x) \frac{\partial \Psi}{\partial y}+\frac{\partial \Psi}{\partial x}=0 \quad \text { in } y=b(x) .
$$

This is equivalent to

$$
\frac{\partial \Psi}{\partial \tau}=0 \quad \text { in } y=b(x)
$$

In the same way, (2.5) gives

$$
\frac{\partial \Psi}{\partial \tau}=0 \quad \text { in } y=1+\gamma(x)
$$

We deduce that $\Psi$ is constant in $y=b(x)$ and $y=1+\gamma(x)$.

Thanks to the condition at infinity, we evaluate the constant which appears in (2.13) and the values of $\Psi$ at the bound of $\Omega_{b}^{\gamma}$. In fact, at infinity we have

$$
\lim _{|x| \rightarrow \infty} \Psi(x, y)=y+k
$$

The function $\Psi$ is a stream function then we can choose $k=0$. Hence

$$
\lim _{|x| \rightarrow \infty} \Psi(x, y)=y .
$$


Replacing these limits in (2.13) we obtain

$$
\frac{F^{2}}{2}+1=c
$$

Moreover, we deduce from (3.7) that

$$
\begin{array}{ll}
\Psi=0 & \text { in } y=b(x), \\
\Psi=1 & \text { in } y=1+\gamma(x) .
\end{array}
$$

Then the stream function $\Psi$ verifies

$$
\begin{gathered}
\Delta \Psi=0 \quad \text { in } \Omega_{b}^{\gamma}, \\
\Psi=0 \quad \text { in } y=b(x), \\
\Psi=1 \quad \text { in } y=1+\gamma(x), \\
\lim _{|x| \rightarrow \infty} \Psi(x, y)=y, \\
\frac{F^{2}}{2}|\nabla \Psi|^{2}(x, 1+\gamma(x))+\gamma(x)=\frac{F^{2}}{2} .
\end{gathered}
$$

Taking into account condition (3.11), we can write

$$
\Psi=y+\psi,
$$

where $\psi$ is the perturbation of the stream function.

Problems (3.10), (3.11), and (3.12) will be written as

$$
\begin{array}{cl}
\Delta \psi=0 & \text { in } \Omega_{b}^{\gamma}, \\
\psi=-b(x) & \text { in } y=b(x), \\
\psi=-\gamma(x) \quad & \text { in } y=1+\gamma(x), \\
& \lim _{|x| \rightarrow \infty} \psi(x, y)=0, \\
\frac{F^{2}}{2}\left(|\nabla \psi|^{2}+2 \frac{\partial \psi}{\partial y}+1\right)+\gamma(x)=\frac{F^{2}}{2} \quad \text { in } y=1+\gamma(x) .
\end{array}
$$

\section{Solution of the free surface problem}

We transform (3.16) in

$$
\gamma(x)=-\frac{F^{2}}{2}\left[|\nabla \psi|^{2}(x, 1+\gamma(x))+2 \frac{\partial \psi}{\partial y}(x, 1+\gamma(x))\right]
$$

and we put

$$
T(b, \gamma)=-\frac{F^{2}}{2}\left[|\nabla \psi|^{2}(x, 1+\gamma(x))+2 \frac{\partial \psi}{\partial y}(x, 1+\gamma(x))\right]
$$


The problem can be formulated as follows: given a function $y=b(x)$ which represents an obstacle, find a function $\gamma: \mathbb{R} \rightarrow \mathbb{R}$ (free surface) such that $T(b, \gamma)=\gamma$ with $\psi$ solution of problems (3.14) and (3.15).

This is equivalent to solving the equation

$$
T_{1}(b, \gamma)=\gamma-T(b, \gamma)=0 \quad \text { for a fixed } b .
$$

For $b=\gamma=0, \psi=0$ verifies (3.14), (3.15), and (3.16). So $T(0,0)=0$ and $T_{1}(0,0)=0$. To solve $T_{1}(b, \gamma)=0$, we use the implicit function theorem at a neighbourhood of $(b, \gamma)=(0,0)$.

Consider the change of variables

$$
\tilde{x}=x, \quad \tilde{y}=\frac{y-b(x)}{1+\gamma(x)-b(x)}
$$

we transform the domain $\Omega_{b}^{\gamma}$ in the following infinite strip $Q$ :

$$
Q=\left\{(x, y) \in \mathbb{R}^{2} \mid-\infty<x<+\infty, 0<y<1\right\} .
$$

We put $\psi(x, y)=\tilde{\psi}(\tilde{x}, \tilde{y})$ then $\tilde{\psi}$ verifies

$$
\begin{gathered}
\Delta \tilde{\psi}+\mathscr{P}_{b}^{\gamma} \tilde{\psi}=0 \quad \text { in } Q, \\
\tilde{\psi}(\tilde{x}, 0)=-b(\tilde{x}), \quad \tilde{\psi}(\tilde{x}, 1)=-\gamma(\tilde{x}), \quad \tilde{x} \in \mathbb{R}
\end{gathered}
$$

$\mathscr{P}_{b}^{\gamma}$ is an operator defined by

$$
\mathscr{P}_{b}^{\gamma}=a_{1} \frac{\partial^{2}}{\partial \tilde{x} \partial \tilde{y}}+a_{2} \frac{\partial^{2}}{\partial \tilde{y}^{2}}+a_{3} \frac{\partial}{\partial \tilde{y}},
$$

where

$$
\begin{gathered}
a_{1}=\frac{\tilde{y}\left(b^{\prime}-\gamma^{\prime}\right)-b^{\prime}}{1+\gamma-b}, \quad a_{2}=\left(\frac{a_{1}}{2}\right)^{2}-1+\frac{1}{(1+\gamma-b)^{2}}, \\
a_{3}=\frac{-1}{1+\gamma-b}\left[b^{\prime \prime}+\tilde{y}\left(\gamma^{\prime \prime}-b^{\prime \prime}\right)\right]+\frac{2}{(1+\gamma-b)^{2}}\left(\gamma^{\prime}-b^{\prime}\right)\left[b^{\prime}+\tilde{y}\left(\gamma^{\prime}-b^{\prime}\right)\right] .
\end{gathered}
$$

The symbols ' and " denote, respectively, the first and second derivative.

The gradient operator becomes

$$
\tilde{\nabla}_{b, \gamma}=\left(\begin{array}{c}
\frac{\partial}{\partial \tilde{x}}+\frac{-b^{\prime}-\tilde{y}\left(\gamma^{\prime}-b^{\prime}\right)}{(1+\gamma-b)^{2}} \frac{\partial}{\partial \tilde{y}} \\
\frac{1}{1+\gamma-b} \frac{\partial}{\partial \tilde{y}}
\end{array}\right) .
$$

Equation (4.1) will be written

$$
\gamma(\tilde{x})=\frac{-F^{2}}{2}\left[\left|\tilde{\nabla}_{b, \gamma} \tilde{\psi}\right|^{2}(\tilde{x}, 1)+\frac{2}{1+\gamma-b} \frac{\partial \tilde{\psi}}{\partial \tilde{y}}(\tilde{x}, 1)\right],
$$


where $b$ is given and $\gamma$ is searched in the space

$$
B_{c}^{2, \lambda}(\mathbb{R})=\left\{v \in C^{2, \lambda}(\mathbb{R}) \sum_{0 \leq k \leq 2} \sup _{x \in \mathbb{R}} e^{c|x|}\left|D_{x}^{k} v(x)\right|<\infty\right\}
$$

and $\tilde{\psi}$ in the space

$$
B_{c}^{2, \lambda}(\bar{Q})=\left\{v \in C^{2, \lambda}(\bar{Q}) \sup _{k+l \leq 2} \sup _{(\tilde{x}, \tilde{y}) \in Q} e^{c|\tilde{x}|}\left|D_{\tilde{x}}^{k} D_{\tilde{y}}^{l} v\right|<\infty\right\},
$$

where $0<\lambda<1$ and $c>0$ [1].

The choice of these spaces will appear evident later.

Remark 4.1. (i) The space $B_{c}^{m, \lambda}(\bar{Q})$ defined by

$$
B_{c}^{m, \lambda}(\bar{Q})=\left\{v \in C^{m, \lambda}(\bar{Q}) \sup _{k+l \leq m} \sup _{(\tilde{x}, \tilde{y}) \in Q} e^{c|\tilde{x}|}\left|D_{\tilde{x}}^{k} D_{\tilde{y}}^{l} v\right|<\infty\right\}
$$

equipped with the norm

$$
\begin{aligned}
\|v\|_{m, c, \lambda}= & \sum_{k+l \leq m} \sup _{(\tilde{x}, \tilde{y}) \in Q} e^{c|\tilde{x}|}\left|D_{\tilde{x}}^{k} D_{\tilde{y}}^{l} v\right| \\
& +\sup _{k+l=m} \sup _{(\tilde{x}, \tilde{y}) \neq\left(\tilde{x}^{\prime}, \tilde{y}^{\prime}\right)} \frac{\left|D_{\tilde{x}}^{k} D_{\tilde{y}}^{l} v(\tilde{x}, \tilde{y})-D_{\tilde{x}}^{k} D_{\tilde{y}}^{l} v\left(\tilde{x^{\prime}}, \tilde{y^{\prime}}\right)\right|}{\left[\left(\tilde{x}-\tilde{x^{\prime}}\right)^{2}+\left(\tilde{y}-\tilde{y^{\prime}}\right)^{2}\right]^{\lambda / 2}}
\end{aligned}
$$

is a Banach algebra.

(ii) The space $B_{c}^{m, \lambda}(\mathbb{R})$ defined by

$$
B_{c}^{m, \lambda}(\mathbb{R})=\left\{v \in C^{m, \lambda}(\mathbb{R}) \sum_{0 \leq k \leq m} \sup _{x \in \mathbb{R}} e^{c|x|}\left|D_{x}^{k} v(x)\right|<\infty\right\}
$$

equipped with the norm

$$
\|v\|_{m, c, \lambda}=\sum_{0 \leq k \leq m} \sup _{x \in \mathbb{R}} e^{c|x|}\left|D_{x}^{k} v\right|+\sup _{\substack{\left(x, x^{\prime}\right) \in \mathbb{R}^{2} \\ x \neq x^{\prime}}} \frac{\left|D_{x}^{m} v(x)-D_{x}^{m} v\left(x^{\prime}\right)\right|}{\left|x-x^{\prime}\right|^{\lambda}}
$$

is also a Banach algebra.

Now we are able to state the main result of this section.

THEOREM 4.2. There exists $\tilde{c}>0, K<1$ such that for all $\lambda, 0<\lambda<1$, and all $c, 0<c<\tilde{c}$, there exists a neighbourhood $\mathscr{V}$ of zero in $B_{c}^{2, \lambda}(\mathbb{R})$ such that for all $F \in] 0, K$ [ problems (3.14), (3.15), and (3.16) have a unique solution $\psi$ such that $\tilde{\psi}$ belongs to $B_{c}^{2, \lambda}(\bar{Q})$, and there exists a mapping $g$ of class $\mathscr{C}^{1}$ such that $\gamma=g(b)$. 
This theorem is equivalent to the following one.

THEOREM 4.3. There exists $\tilde{c}>0, K<1$, and an open ball $\mathscr{B}$ of radius $r_{0}$ centered at the origin of $B_{c}^{2, \lambda}(\mathbb{R}) \times B_{c}^{2, \lambda}(\mathbb{R})$ where $\left.c \in\right] 0, \tilde{c}[$, and $0<\lambda<1$, there exists a neighbourhood $\mathscr{V}_{b}$ of zero in $B_{c}^{2, \lambda}(\mathbb{R})$, there exists a mapping

$$
g: \mathscr{V}_{b} \longrightarrow B_{c}^{2, \lambda}(\mathbb{R})
$$

of class $\mathscr{C}^{1}$, such that for all $\left.F \in\right] 0, K\left[,\left\{\right.\right.$ for all $\left.(b, \gamma) \in \mathscr{B}, T_{1}(b, \gamma)=0\right\} \Leftrightarrow$ $\left\{b \in \mathscr{V}_{b}, \gamma=g(b)\right\}$.

Proof. In the next subsections, we will verify the hypothesis of the implicit function theorem.

4.1. Differentiability of the operator $T_{1}$ with respect to $(b, \gamma)$. We have $T_{1}(b, \gamma)=\gamma-T(b, \gamma)$; to show the differentiability of $T_{1}$ with respect to $b$ and $\gamma$, it suffices to study the differentiability of $T$ with respect to $b$ and $\gamma$. For this we use the following results.

THEOREM 4.4. There exists $\tilde{c}>0$ such that for all $c \in] 0, \tilde{c}[$ and $\lambda \in] 0,1[$, there exists an open ball $\mathscr{B}$ of radius $r_{0}>0$, centered at the origin in $B_{c}^{2, \lambda}(\mathbb{R}) \times$ $B_{c}^{2, \lambda}(\mathbb{R})$ such that whenever $(b, \gamma) \in \mathscr{B}$, the following statements hold:

(a) the problem

$$
\begin{gathered}
\Delta \psi=0 \quad \text { in } \Omega_{b}^{\gamma}, \\
\psi(x, 1+\gamma(x))=-\gamma(x), \quad \psi(x, b(x))=-b(x), \quad x \in \mathbb{R}
\end{gathered}
$$

has a unique solution $\psi$ such that $\tilde{\psi}$, the transform of $\psi$ by (4.4) is in $B_{c}^{2, \lambda}(\bar{Q})$;

(b) the mapping $S:(b, \gamma) \mapsto \tilde{\psi}$ is continuously differentiable from $\mathscr{B}$ into $B_{c}^{2, \lambda}(\bar{Q})$.

To prove this theorem, we use the following proposition which is proved in the annex.

Proposition 4.5. Let the boundary value problem

$$
\begin{gathered}
\Delta v=b_{1} \quad \text { in } Q, \\
v(\tilde{x}, 1)=b_{2}(\tilde{x}), \quad v(\tilde{x}, 0)=b_{3}(\tilde{x}), \quad \tilde{x} \in \mathbb{R},
\end{gathered}
$$

where $\left(b_{1}, b_{2}, b_{3}\right) \in B_{c}^{0, \lambda}(\bar{Q}) \times B_{c}^{2, \lambda}(\mathbb{R}) \times B_{c}^{2, \lambda}(\mathbb{R})$, then there exists $\tilde{c}>0$ such that whenever $0<c<\tilde{c}$, problem (4.19) has a unique solution $v \in B_{c}^{2, \lambda}(\bar{Q})$. Furthermore the solution map is a topological isomorphism between the corresponding spaces. 


\section{Proof of Theorem 4.4}

Proof of $(a)$. Denote by $\mathscr{A}_{b}^{\gamma}$ the linear operator defined by

$$
\begin{gathered}
\mathscr{A}_{b}^{\gamma}: B_{c}^{2, \lambda}(\bar{Q}) \longrightarrow B_{c}^{0, \lambda}(\bar{Q}) \times B_{c}^{2, \lambda}(\mathbb{R}) \times B_{c}^{2, \lambda}(\mathbb{R})=\mathscr{Y}, \\
v \longmapsto\left(\Delta v+\mathscr{P}_{b}^{\gamma} v, v(\cdot, 0), v(\cdot, 1)\right),
\end{gathered}
$$

and $\mathscr{A}=\mathscr{A}_{0}^{0}$. We will verify that

$$
\left\|\left(\mathscr{A}-\mathscr{A}_{b}^{\gamma}\right) v\right\|_{\mathscr{y}} \leq L\left(\|b\|_{B_{c}^{2, \lambda}(\mathbb{R})},\|\gamma\|_{B_{c}^{2, \lambda}(\mathbb{R})}\right) \cdot\|v\|_{B_{c}^{2, \lambda}(\bar{Q})}
$$

where $L(\cdot, \cdot)$ is a continuous function on $\mathbb{R}^{2}$ verifying $L(0,0)=0$. We have $\left(\mathscr{A}-\mathscr{A}_{b}^{\gamma}\right) v=\left(-\mathscr{P}_{b}^{\gamma} v, 0,0\right)$.

Then

$$
\begin{aligned}
\left\|\left(\mathscr{A}-\mathscr{A}_{b}^{\gamma}\right) v\right\|_{\mathscr{Y}} & =\left\|\mathscr{P}_{b}^{\gamma} v\right\|_{B_{c}^{0, \lambda}(\bar{Q})} \\
& =\left\|a_{1} \frac{\partial^{2}}{\partial \tilde{x} \partial \tilde{y}}+a_{2} \frac{\partial^{2}}{\partial \tilde{y}^{2}}+a_{3} \frac{\partial}{\partial \tilde{y}}\right\|_{B_{c}^{0, \lambda}(\bar{Q})} .
\end{aligned}
$$

We need the following lemma which is evident to prove.

Lemma 4.6. Let $(b, \gamma) \in B_{c}^{2, \lambda}(\mathbb{R}) \times B_{c}^{2, \lambda}(\mathbb{R})$. We have $\left(a_{1}, a_{2}, a_{3}\right) \in\left(B_{c}^{0, \lambda}(\bar{Q})\right)^{3}$ furthermore, $\left\|a_{i}\right\|_{B_{c}^{0, \lambda}(\bar{Q})} \leq L_{i}\left(\|b\|_{B_{c}^{2, \lambda}(\mathbb{R})},\|\gamma\|_{B_{c}^{2, \lambda}(\mathbb{R})}\right), 1 \leq i \leq 3$ where $L_{i}(\cdot, \cdot)$ is a continuous function verifying $L_{i}(0,0)=0$.

Then we have

$$
\begin{aligned}
\left\|\mathscr{P}_{b}^{\gamma} v\right\|_{B_{c}^{0, \lambda}(\bar{Q})} & \leq\left\|a_{1} \frac{\partial^{2} v}{\partial \tilde{x} \partial \tilde{y}}\right\|_{B_{c}^{0, \lambda}(\bar{Q})}+\left\|a_{2} \frac{\partial^{2} v}{\partial \tilde{y}^{2}}\right\|_{B_{c}^{0, \lambda}(\bar{Q})}+\left\|a_{3} \frac{\partial v}{\partial \tilde{y}}\right\|_{B_{c}^{0, \lambda}(\bar{Q})} \\
& \leq\left(\left\|a_{1}\right\|_{B_{c}^{0, \lambda}(\bar{Q})}+\left\|a_{2}\right\|_{B_{c}^{0, \lambda}(\bar{Q})}+\left\|a_{3}\right\|_{B_{c}^{0, \lambda}(\bar{Q})}\right)\|v\|_{B_{c}^{2, \lambda}(\bar{Q})}
\end{aligned}
$$

and using the last lemma we obtain

$$
\begin{aligned}
\left\|\mathscr{P}_{b}^{\gamma} v\right\|_{B_{c}^{0, \lambda}(\bar{Q})} \leq & \left(L_{1}\left(\|b\|_{B_{c}^{2, \lambda}(\mathbb{R})},\|\gamma\|_{B_{c}^{2, \lambda}(\mathbb{R})}\right)+L_{2}\left(\|b\|_{B_{c}^{2, \lambda}(\mathbb{R})},\|\gamma\|_{B_{c}^{2, \lambda}(\mathbb{R})}\right)\right. \\
& \left.+L_{3}\left(\|b\|_{B_{c}^{2, \lambda}(\mathbb{R})},\|\gamma\|_{B_{c}^{2, \lambda}(\mathbb{R})}\right)\right)\|v\|_{B_{c}^{2, \lambda}(\bar{Q})} .
\end{aligned}
$$

So

$$
\left\|\left(\mathscr{A}-\mathscr{A}_{b}^{\gamma}\right) v\right\|_{\mathscr{y}} \leq L\left(\|b\|_{B_{c}^{2, \lambda}(\mathbb{R})},\|\gamma\|_{B_{c}^{2, \lambda}(\mathbb{R})}\right)\|v\|_{B_{c}^{2, \lambda}(\bar{Q})},
$$

where $L(\cdot, \cdot)$ is a continuous function verifying $L(0,0)=0$. 
The operator $\mathscr{A}$ being an isomorphism, this shows that $\mathscr{A}_{b}^{\gamma}$ is also an isomorphism for small $b$ and $\gamma$.

Thus the problem

$$
\begin{gathered}
\Delta \tilde{\psi}+\mathscr{P}_{b}^{\gamma} \tilde{\psi}=0 \quad \text { in } Q, \\
\tilde{\psi}(\tilde{x}, 0)=-b(\tilde{x}), \quad \tilde{\psi}(\tilde{x}, 1)=-\gamma(\tilde{x}), \quad \tilde{x} \in \mathbb{R},
\end{gathered}
$$

has a unique solution in $B_{c}^{2, \lambda}(\bar{Q})$. This gives the proof of Theorem 4.4(a).

Now we will prove (b), that is, the application

$$
\begin{gathered}
S: \mathscr{B}\left(0, r_{0}\right) \subset B_{c}^{2, \lambda}(\mathbb{R}) \times B_{c}^{2, \lambda}(\mathbb{R}) \longrightarrow B_{c}^{2, \lambda}(\bar{Q}) \\
(b, \gamma) \longmapsto \tilde{\psi}
\end{gathered}
$$

is continuously differentiable.

We define $S_{1}$ and $S_{2}$ as follows:

$$
\begin{aligned}
S_{1}: B\left(0, r_{0}\right) & \longrightarrow \mathscr{L}\left(B_{c}^{2, \lambda}(\bar{Q}), y\right), \\
(b, \gamma) & \longmapsto \mathscr{A}_{b}^{\gamma} \\
S_{2}: \operatorname{Isom}\left(B_{c}^{2, \lambda}(\bar{Q}), \text {, }\right) & \longrightarrow \operatorname{Isom}\left(\mathscr{Y}, B_{c}^{2, \lambda}(\bar{Q})\right), \\
L & \longmapsto L^{-1}
\end{aligned}
$$

and we put

$$
\mathscr{F}(b, \gamma)=(0,-b(\tilde{x}),-\gamma(\tilde{x}))=\mathscr{A}_{b}^{\gamma} \tilde{\psi} \quad \text { in } \mathscr{Y} .
$$

We have

$$
\begin{gathered}
S_{2} \circ S_{1}(b, \gamma)=\left(\mathscr{A}_{b}^{\gamma}\right)^{-1} \text { with }(b, \gamma) \in B\left(0, r_{0}\right), \\
S(b, \gamma)=\left(S_{2} \circ S_{1}(b, \gamma)\right) \mathscr{F}(b, \gamma)=\left(\mathscr{A}_{b}^{\gamma}\right)^{-1}\left(\mathscr{A}_{b}^{\gamma} \tilde{\psi}\right)=\tilde{\psi} .
\end{gathered}
$$

The differentiability of $\tilde{\psi}$ is given by the differentiability of $S_{1}, S_{2}$, and $\mathscr{F}(b, \gamma)$. It is evident that $\mathscr{F}(b, \gamma)$ is continuously differentiable with respect to $b$ and $\gamma$. $S_{2}$ is a $\mathscr{C}^{\infty}$ operator. It remains to prove the continuous differentiability of $S_{1}$.

We have

$$
S_{1}:(b, \gamma) \longrightarrow \mathscr{A}_{b}^{\gamma}=\Delta+a_{1} \frac{\partial^{2}}{\partial \tilde{x} \partial \tilde{y}}+a_{2} \frac{\partial^{2}}{\partial \tilde{y}^{2}}+a_{3} \frac{\partial}{\partial \tilde{y}} .
$$

It is sufficient to prove that $a_{i}, 1 \leq i \leq 3$, which are rational functions in $b, \gamma$, $b^{\prime}, \gamma^{\prime}, b^{\prime \prime}, \gamma^{\prime \prime}$, are continuously differentiable with respect to $b$ and $\gamma$. For this we use the next lemma which is evident to prove [1]. 
LEMMA 4.7. Let $p$ be a rational function of $k$ variables which is devoid of poles in a neighbourhood of the origin in $\mathbb{R}^{k}$ ( $k$ a positive integer) such that $p(0)=0$. Then the mapping

$$
\begin{gathered}
P: \Pi_{1 \leq i \leq k} B_{c}^{n_{i}, \lambda}(\mathbb{R}) \longrightarrow B_{c}^{n_{0}, \lambda}(\mathbb{R}), \\
\left(g_{1}, \ldots, g_{k}\right) \longmapsto P\left(g_{1}, \ldots, g_{k}\right),
\end{gathered}
$$

where $n_{i} \in \mathbb{N}, 1 \leq i \leq k, n_{0}=\min \left\{n_{i}, 1 \leq i \leq k\right\}$ is continuously differentiable in a neighbourhood of the origin in $\Pi_{1 \leq i \leq k} B_{c}^{n_{i}, \lambda}(\mathbb{R})$.

The coefficients $a_{1}, a_{2}$, and $a_{3}$ verify the hypothesis of Lemma 4.7, then they are continuously differentiable with respect to $b$ and $\gamma$. This gives continuous differentiability of $S_{1}$ with respect to $b$ and $\gamma$. Then Theorem 4.4 is proved.

In the new variables $\tilde{x}, \tilde{y}$, the operator $T(b, \gamma)$ takes the form

$$
\begin{aligned}
T(b, \gamma)=-\frac{F^{2}}{2}\left\{\left[\frac{\partial \tilde{\psi}}{\partial \tilde{x}}(\tilde{x}, 1)-\frac{\gamma^{\prime}}{(1+\gamma-b)^{2}} \frac{\partial \tilde{\psi}}{\partial \tilde{y}}(\tilde{x}, 1)\right]^{2}\right. \\
\left.+\frac{1}{(1+\gamma-b)^{2}}\left(\frac{\partial \tilde{\psi}}{\partial \tilde{y}}(\tilde{x}, 1)\right)^{2}+\frac{2}{1+\gamma-b} \frac{\partial \tilde{\psi}}{\partial \tilde{y}}(\tilde{x}, 1)\right\} \\
=-\frac{F^{2}}{2}\left\{\left[\frac{\partial \tilde{\psi}}{\partial \tilde{x}}(\tilde{x}, 1)+\lambda_{1}(b, \gamma) \frac{\partial \tilde{\psi}}{\partial \tilde{y}}(\tilde{x}, 1)\right]^{2}\right. \\
\left.+\lambda_{2}^{2}(b, \gamma)\left(\frac{\partial \tilde{\psi}}{\partial \tilde{y}}(\tilde{x}, 1)\right)^{2}+2 \lambda_{2}(b, \gamma) \frac{\partial \tilde{\psi}}{\partial \tilde{y}}(\tilde{x}, 1)\right\}
\end{aligned}
$$

with

$$
\lambda_{1}(b, \gamma)=-\frac{\gamma^{\prime}}{(1+\gamma-b)^{2}} ; \quad \lambda_{2}(b, \gamma)=\frac{1}{1+\gamma-b}
$$

THEOREM 4.8. Under the hypothesis of Theorem 4.4 the operator $T$ is continuously Gâteaux differentiable on $B$.

Proof. We have shown that $\tilde{\psi}$ is continuously differentiable with respect to $b$ and $\gamma$. Moreover, it is evident that $\lambda_{1}(b, \gamma)$ and $\lambda_{2}(b, \gamma)$ are continuously differentiable with respect to $b$ and $\gamma$. We deduce that $T(b, \gamma)$ is continuously Gâteaux differentiable with respect to $b$ and $\gamma$. Then $T_{1}$ is continuously differentiable on $B$.

4.2. Expression of $\left(\partial T_{1} / \partial \gamma\right)(0,0)$. In the last subsection, we have seen that $\tilde{\psi}$ is the solution of the problem

$$
\begin{gathered}
\Delta \tilde{\psi}+\mathscr{P}_{b}^{\gamma} \tilde{\psi}=0 \quad \text { in } Q, \\
\tilde{\psi}(\tilde{x}, 0)=-b(\tilde{x}), \quad \tilde{\psi}(\tilde{x}, 1)=-\gamma(\tilde{x}), \quad \tilde{x} \in \mathbb{R} .
\end{gathered}
$$


We have $\tilde{\psi}_{\mid b=\gamma=0}=0$ in $Q$ and $\mathscr{P}_{0}^{0} \tilde{\psi}=0$.

Let $h \in B_{c}^{2, \lambda}(\mathbb{R})$. We put $b=0$ in system (4.35), we derive with respect to $\gamma$ in the direction $h$ and we evaluate the derivative at $\gamma=0$. We put

$$
w=\left.\frac{\partial \tilde{\psi}}{\partial \gamma}\right|_{b=\gamma=0} .
$$

We obtain

$$
\begin{gathered}
\Delta w+\mathscr{P}_{0}^{0} w+\frac{\partial}{\partial \gamma}\left(\mathscr{P}_{0}^{\gamma}\right)_{\mid \gamma=0} \tilde{\psi}_{\mid b=\gamma=0} \cdot h=0 \quad \text { in } Q, \\
w(\tilde{x}, 0)=0, \quad w(\tilde{x}, 1)=-h(\tilde{x}), \quad \tilde{x} \in \mathbb{R}
\end{gathered}
$$

and we get the following result.

Theorem 4.9. Let $h \in B_{c}^{2, \lambda}(\mathbb{R})$ and $w=w(h)=(\partial \tilde{\psi} / \partial \gamma)(\cdot, 1)_{\mid b=\gamma=0} \cdot h$. Then $w(h)$ is the unique solution of the problem

$$
\begin{gathered}
\Delta w=0 \quad \text { in } Q, \\
w(\tilde{x}, 0)=0, \quad w(\tilde{x}, 1)=-h(\tilde{x}), \quad \tilde{x} \in \mathbb{R}
\end{gathered}
$$

and satisfies

$$
\|w(h)\|_{B_{c}^{2, \lambda}(\bar{Q})} \leq k\|h\|_{B_{c}^{2, \lambda}(\mathbb{R})}, \quad k>1 .
$$

Proof. For the existence and uniqueness, we use Proposition 4.5. Now to prove inequality (4.39), we need the next lemma proved in the annex.

Lemma 4.10. Let $h$ be in the space $B_{c}^{2, \lambda}(\mathbb{R})$. Then the function $(\tilde{x}, \tilde{y}) \mapsto$ $h(\tilde{x}) \tilde{y}$ is in the space $B_{c}^{2, \lambda}(\bar{Q})$ and verify $\|h(\tilde{x}) \tilde{y}\|_{B_{c}^{2, \lambda}(\bar{Q})} \leq k\|h\|_{B_{c}^{2, \lambda}(\mathbb{R})}$, where $k>1$.

Now we are able to establish relation (4.39). We can write

$$
w(\tilde{x}, \tilde{y})=u(\tilde{x}, \tilde{y})-h(\tilde{x}) \tilde{y},
$$

where $u$ is the solution of the problem

$$
\begin{gathered}
\Delta u=h^{\prime \prime}(\tilde{x}) \tilde{y} \quad \text { in } Q, \\
u(\tilde{x}, 0)=0, \quad u(\tilde{x}, 1)=0, \quad \tilde{x} \in \mathbb{R} .
\end{gathered}
$$

From Proposition 4.5 we have

$$
\begin{aligned}
\|u\|_{B_{c}^{2, \lambda}\left(\sum\right)} & \leq k_{1}\|\Delta u\|_{B_{c}^{0, \lambda}\left(\sum\right)}, \quad k_{1}>1 \text { (evident) } \\
& \leq k_{1}\left\|h^{\prime \prime}(\tilde{x}) \tilde{y}\right\|_{B_{c}^{0, \lambda}(\bar{Q})} \\
& \leq k_{1} k_{2}\|h\|_{B_{c}^{2, \lambda}(\mathbb{R})}, \quad k_{2}>1(\text { Lemma } 4.10) \\
& \leq k_{3}\|h\|_{B_{c}^{2, \lambda}(\mathbb{R})}, \quad \text { where } k_{3}=k_{1} k_{2} .
\end{aligned}
$$


Using Lemma 4.10 we obtain

$$
\begin{aligned}
\|w(h)\|_{B_{c}^{2, \lambda}(\bar{Q})} & \leq k_{3}\|h\|_{B_{c}^{2, \lambda}(\mathbb{R})}+k_{4}\|h\|_{B_{c}^{2, \lambda}(\mathbb{R})} \\
& \leq k\|h\|_{B_{c}^{2, \lambda}(\mathbb{R})}, \quad k>1,
\end{aligned}
$$

where $k=k_{3}+k_{4}$. Now we can evaluate $\left(\partial T_{1} / \partial \gamma\right)(0,0)$. We have

$$
\frac{\partial T_{1}}{\partial \gamma}(0,0) \cdot h=\left[\mathrm{Id}-\frac{\partial T}{\partial \gamma}(0,0)\right] \cdot h, \quad h \in B_{c}^{2, \lambda}(\mathbb{R}),
$$

where Id is the identity mapping of $B_{c}^{2, \lambda}(\mathbb{R})$.

We calculate $(\partial T / \partial \gamma)(0,0) \cdot h$

$$
\begin{aligned}
T(0, \gamma)=-\frac{F^{2}}{2}\{ & {\left[\frac{\partial \tilde{\psi}}{\partial \tilde{x}}(\cdot, 1)-\frac{\gamma^{\prime}}{(1+\gamma-b)^{2}} \frac{\partial \tilde{\psi}}{\partial \tilde{y}}(\cdot, 1)\right]^{2} } \\
& \left.+\frac{1}{(1+\gamma-b)^{2}}\left(\frac{\partial \tilde{\psi}}{\partial \tilde{y}}(\cdot, 1)\right)^{2}+\frac{2}{1+\gamma-b} \frac{\partial \tilde{\psi}}{\partial \tilde{y}}(\cdot, 1)\right\}_{\mid b=0} .
\end{aligned}
$$

We derive with respect to $\gamma$ in the direction $h$ at $\gamma=0$

$$
\begin{aligned}
\frac{\partial T}{\partial \gamma}(0,0) \cdot h=-\frac{F^{2}}{2}\{ & 2\left[\frac{\partial \tilde{\psi}}{\partial \tilde{x}}(\cdot, 1)-\frac{\gamma^{\prime}}{(1+\gamma)^{2}} \frac{\partial \tilde{\psi}}{\partial \tilde{y}}(\cdot, 1)\right]_{\mid b=\gamma=0} \\
& \times \frac{\partial}{\partial \gamma}\left[\frac{\partial \tilde{\psi}}{\partial \tilde{x}}(\cdot, 1)-\frac{\gamma^{\prime}}{(1+\gamma)^{2}} \frac{\partial \tilde{\psi}}{\partial \tilde{y}}(\cdot, 1)\right] \cdot h \\
& +\frac{\partial}{\partial \gamma}\left(\frac{\partial \tilde{\psi}}{\partial \tilde{y}}\right)^{2}(\cdot, 1)_{\mid b=\gamma=0} \cdot h-2\left[\frac{\partial \tilde{\psi}}{\partial \tilde{y}}_{\mid b=\gamma=0}(\cdot, 1)\right]^{2} \cdot h \\
& \left.-2\left[\frac{\partial \tilde{\psi}}{\partial \tilde{y}}_{\mid b=\gamma=0}(\cdot, 1)\right] \cdot h+2 \frac{\partial}{\partial \gamma}\left(\frac{\partial \tilde{\psi}}{\partial \tilde{y}}(\cdot, 1)_{\mid b=\gamma=0}\right) \cdot h\right\}
\end{aligned}
$$

which gives

$$
\begin{aligned}
\frac{\partial T}{\partial \gamma}(0,0) \cdot h & =-F^{2}\left\{\frac{\partial}{\partial \gamma}\left(\frac{\partial \tilde{\psi}}{\partial \tilde{y}}(\cdot, 1)_{\mid b=\gamma=0}\right) \cdot h\right\} \\
& =-F^{2}\left\{\frac{\partial}{\partial \tilde{y}}\left(\frac{\partial \tilde{\psi}}{\partial \gamma}(\cdot, 1)_{\mid b=\gamma=0}\right) \cdot h\right\} \\
& =-F^{2} \frac{\partial}{\partial \tilde{y}}(w(h)) .
\end{aligned}
$$

We replace $(\partial T / \partial \gamma)(0,0) \cdot h$ by its expression in (4.44) and we find

$$
\frac{\partial T_{1}}{\partial \gamma}(0,0) \cdot h=\left[\operatorname{Id}+F^{2} \frac{\partial w}{\partial \tilde{y}}\right] \cdot h .
$$


4.3. Inversibility of $\left(\partial T_{1} / \partial \gamma\right)(0,0)$. To prove the inversibility of $\left(\partial T_{1} / \partial \gamma\right)$ $(0,0)=\operatorname{Id}+F^{2}(\partial w / \partial \tilde{y})$, it suffices to show that

$$
\left\|F^{2} \frac{\partial w}{\partial \tilde{y}}\right\|_{\mathscr{L}\left(B_{c}^{2, \lambda}(\mathbb{R}), B_{c}^{1, \lambda}(\mathbb{R})\right)}<1 .
$$

We have

$$
\begin{aligned}
& \left\|F^{2} \frac{\partial w}{\partial \tilde{y}}\right\|_{\mathscr{L}\left(B_{c}^{2, \lambda}(\mathbb{R}), B_{c}^{1, \lambda}(\mathbb{R})\right)}=F^{2} \sup _{h \neq 0} \frac{\|\partial w / \partial \tilde{y}\|_{B_{c}^{1, \lambda}(\mathbb{R})}}{\|h\|_{B_{c}^{2, \lambda}(\mathbb{R})}} \\
& \leq F^{2} \sup _{h \neq 0} \frac{\|w(h)\|_{B_{c}^{2, \lambda}(\mathbb{R})}}{\|h\|_{B_{c}^{2, \lambda}(\mathbb{R})}}
\end{aligned}
$$

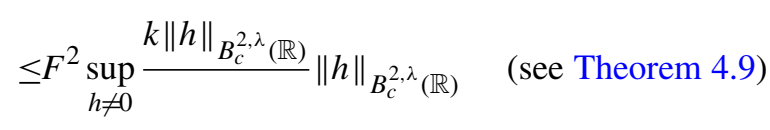

$$
\begin{aligned}
& \leq F^{2} k \text {. }
\end{aligned}
$$

It suffices that $F^{2} k<1$ for the inversibility of $\left(\partial T_{1} / \partial \gamma\right)(0,0)$. Then the inversibility of $\left(\partial T_{1} / \partial \gamma\right)(0,0)$ is obtained for $\left.F \in\right] 0,1 / \sqrt{k}[$. These results achieve the proof of Theorem 4.3 and then the proof of Theorem 4.2.

Remark 4.11. We have a result of existence and uniqueness for Froude numbers $F<1$.

\section{Conclusion}

The conclusion of this work is that we have established a local result of existence and uniqueness of the solution for the values of Froude number in $] 0, k[\subset] 0,1[$. Precisely, for a given small obstacle $b$ in $B_{c}^{2, \lambda}(\mathbb{R})$ there exists a unique function $\gamma$ in $B_{c}^{2, \lambda}(\mathbb{R})$ which describes the perturbation of the free surface. The result established here does not exclude the existence of solutions in other spaces. In [5], it is said that there is no uniqueness of the solution when $F<1$. This result has been confirmed numerically in [5] where moreover the existence and uniqueness of the solution are established after linearization of the equations. In our paper, without linearization, we have solved the problem of existence and uniqueness using the implicit function theorem in subspaces of Hölder spaces.

\section{Annex}

Proof of Proposition 4.5.

Consider the homogeneous problem

$$
\begin{gathered}
\Delta v=f_{1} \quad \text { in } Q, \\
v(\cdot, 1)=v(\cdot, 0)=0 \quad \text { in } \mathbb{R} .
\end{gathered}
$$


First of all, $f_{1}$ belongs to $B_{c}^{0, \lambda}(\bar{Q})$ then $f_{1}$ obviously belongs to $L^{2}(Q)$. So there exists a weak solution $v \in H_{0}^{1}(Q)$. In fact $v \in H^{2}(Q) \cap H_{0}^{1}(Q)$. Because $f_{1} \in B_{c}^{0, \lambda}(\bar{Q})$ we conclude that $v$ is a classical solution in $\mathscr{C}^{2, \lambda}(\bar{Q})$ (see [4]).

To prove that $v \in B_{c}^{2, \lambda}(\bar{Q})$, we must show that

$$
\sup _{k+l \leq 2} \sup _{Q} e^{c|x|}\left|D_{x}^{k} D_{y}^{l} v\right|<\infty
$$

For this we use the result established in [2] where we replace the operator $L$ by $-\Delta$. We conclude that there exists $\tilde{c}>0$ such that for each $c \in] 0, \tilde{c}[$, problem (5.1) has a unique solution in $B_{c}^{2, \lambda}(\bar{Q})$ when $f_{1}$ is given in $B_{c}^{0, \lambda}(\bar{Q})$.

We return to the nonhomogeneous problem

$$
\begin{gathered}
\Delta v=b_{1} \quad \text { in } Q, \\
v(x, 1)=b_{2}(x), \quad v(x, 0)=b_{3}(x), \quad x \in \mathbb{R} .
\end{gathered}
$$

We put $v(x, y)=v_{1}(x, y)+(1-y) b_{3}(x)+y b_{2}(x)$ where $v_{1}$ verifies

$$
\begin{gathered}
\Delta v_{1}=b_{1}-\Delta\left((1-y) b_{3}(x)\right)-\Delta\left(y b_{2}(x)\right)=F(x, y) \quad \text { in } Q, \\
v_{1}(x, 1)=0, \quad v_{1}(x, 0)=0, \quad x \in \mathbb{R} .
\end{gathered}
$$

It is evident that $F \in B_{c}^{0, \lambda}(\bar{Q})$ so $v_{1} \in B_{c}^{2, \lambda}(\bar{Q})$.

We deduce that $v \in B_{c}^{2, \lambda}(\bar{Q})$.

Proof of Lemma 4.10. We put

$$
u(x, y)=h(x) y
$$

The functions $(x, y) \mapsto h(x)$ and $(x, y) \mapsto y$ are in $\mathscr{C}^{2, \lambda}(\bar{Q})$. Then $(x, y) \mapsto$ $h(x) y$ is in $\mathscr{C}^{2, \lambda}(\bar{Q})$.

We also have

$$
\sup _{(x, y) \in Q} \sup _{k+l \leq 2} e^{c|x|}\left|D_{x}^{k} D_{y}^{l} u(x, y)\right|<\infty
$$

Then

$$
u(x, y)=h(x) y \in B_{c}^{2, \lambda}(\bar{Q})
$$


Now we show that

$$
\begin{aligned}
& \|h(x) y\|_{B_{c}^{2, \lambda}(\bar{Q})} \leq k\|h\|_{B_{c}^{2, \lambda}(\mathbb{R})}, \\
& \|h(x) y\|_{B_{c}^{2, \lambda}(\bar{Q})} \\
& =\sum_{k+l \leq 2} \sup _{(x, y) \in \bar{Q}} e^{c|x|}\left|D_{x}^{k} D_{y}^{l}(h(x) y)\right| \\
& +\sup _{k+l=2} \sup _{(x, y) \neq\left(x^{\prime}, y^{\prime}\right)} \frac{\left|D_{x}^{k} D_{y}^{l}(h(x) y)(x, y)-D_{x}^{k} D_{y}^{l}(h(x) y)\left(x^{\prime}, y^{\prime}\right)\right|}{\left[\left(x-x^{\prime}\right)^{2}+\left(y-y^{\prime}\right)^{2}\right]^{\lambda / 2}} \\
& =\sup _{(x, y) \in \bar{Q}} e^{c|x|}|h(x) y|+\sup _{(x, y) \in \bar{Q}} e^{c|x|}\left|h^{\prime}(x) y\right|+\sup _{(x, y) \in \bar{Q}} e^{c|x|}\left|h^{\prime \prime}(x) y\right| \\
& +\sup _{(x, y) \in \bar{Q}} e^{c|x|}|h(x)|+\max \left\{\sup _{(x, y) \neq\left(x^{\prime}, y^{\prime}\right)} \frac{\left|h^{\prime \prime}(x) y-h^{\prime \prime}\left(x^{\prime}\right) y^{\prime}\right|}{\left[\left(x-x^{\prime}\right)^{2}+\left(y-y^{\prime}\right)^{2}\right]^{\lambda / 2}},\right. \\
& \left.\times \sup _{(x, y) \neq\left(x^{\prime}, y^{\prime}\right)} \frac{\left|h^{\prime}(x)-h^{\prime}\left(x^{\prime}\right)\right|}{\left[\left(x-x^{\prime}\right)^{2}+\left(y-y^{\prime}\right)^{2}\right]^{\lambda / 2}}\right\}, \\
& \|h(x) y\|_{B_{c}^{2, \lambda}(\bar{Q})} \leq 2\|h\|_{B_{c}^{2, \lambda}(\mathbb{R})}+\|h\|_{B_{c}^{1, \lambda}(\mathbb{R})} \\
& +\sup _{(x, y) \neq\left(x^{\prime}, y^{\prime}\right)} \frac{\left|h^{\prime \prime}(x) y-h^{\prime \prime}\left(x^{\prime}\right) y^{\prime}\right|}{\left[\left(x-x^{\prime}\right)^{2}+\left(y-y^{\prime}\right)^{2}\right]^{\lambda / 2}} \text {. }
\end{aligned}
$$

We have

$$
\begin{aligned}
& \sup _{(x, y) \neq\left(x^{\prime}, y^{\prime}\right)} \frac{\left|h^{\prime \prime}(x) y-h^{\prime \prime}\left(x^{\prime}\right) y^{\prime}\right|}{\left[\left(x-x^{\prime}\right)^{2}+\left(y-y^{\prime}\right)^{2}\right]^{\lambda / 2}} \\
& \quad \leq \sup _{(x, y) \neq\left(x^{\prime}, y^{\prime}\right)} \frac{\left|h^{\prime \prime}(x) y-h^{\prime \prime}\left(x^{\prime}\right) y+h^{\prime \prime}\left(x^{\prime}\right) y-h^{\prime \prime}\left(x^{\prime}\right) y^{\prime}\right|}{\left[\left(x-x^{\prime}\right)^{2}+\left(y-y^{\prime}\right)^{2}\right]^{\lambda / 2}} \\
& \quad \leq \sup _{x \neq x^{\prime}} \frac{\left|h^{\prime \prime}(x)-h^{\prime \prime}\left(x^{\prime}\right)\right|}{\left|x-x^{\prime}\right|^{\lambda}}+\sup _{(x, y) \neq\left(x^{\prime}, y^{\prime}\right)} \frac{\left|h^{\prime \prime}\left(x^{\prime}\right)\right|\left|y-y^{\prime}\right|}{\left|y-y^{\prime}\right|^{\lambda}} \\
& \leq\|h\|_{B_{c}^{2, \lambda}(\mathbb{R})}+\left|y-y^{\prime}\right|^{1-\lambda}\|h\|_{B_{c}^{2, \lambda}(\mathbb{R})} \\
& \leq 2\|h\|_{B_{c}^{2, \lambda}(\mathbb{R})} .
\end{aligned}
$$

Then we obtain

$$
\|h(x) y\|_{B_{c}^{2, \lambda}(\bar{Q})} \leq(4+c)\|h\|_{B_{c}^{2, \lambda}(\mathbb{R})}
$$


and we have

$$
\|h(x) y\|_{B_{c}^{2, \lambda}(\bar{Q})} \leq k\|h\|_{B_{c}^{2, \lambda}(\mathbb{R})},
$$

where $k>1$.

\section{References}

[1] F. Abergel and J. L. Bona, Mathematical theory for viscous, free-surface flows over a perturbed plane, Department of Mathematics Report AM81, Pennstate, February 1991.

[2] C. J. Amick, On the Dirichlet problem for infinite cylinders and equations with transversely varying coefficients, J. Differential Equations 30 (1978), no. 2, 248279. MR 81g:35030.

[3] M. Bouhadef, Contribution à l'étude des ondes de surface dans un canal. Application à l'écoulement au dessus d'un obstacle immergé, Thèse de doctorat des sciences physiques, Université de Poitiers, U.E.R centre d'études aérodynamiques et thermiques, 1988.

[4] R. Dautray and J.-L. Lions, Analyse Mathématique et Calcul Numérique Pour les Sciences et les Techniques. Vol. 1, Masson, Paris, 1987 (French). MR 88m:00002.

[5] F. Helein, Ecoulement stationnaire dans un canal à fond presque plat, Tech. Report 9205, groupe Hydrodynamique Navale, URA CNRS 853, ENSTA, Centre de l'Yvette 91120 Palaiseau, France, 1992.

[6] A. C. King and M. I. G. Bloor, Free streamline flow over curved topography, Quart. Appl. Math. 48 (1990), no. 2, 281-293. MR 91g:76008. Zbl 0702.76016.

D. Boukari and R. Djouadi: Département d’analyse, Institut de Mathématiques, U.S.T.H.B, BP 32 El Alia Bab, Ezzouar Alger, Algeria

D. Teniou: Département D'analyse, Institut de Mathématiques, U.S.T.H.B, BP 32 El Alia Bab, Ezzouar Alger, Algeria

E-mail address: tenioud@wissal.dz 


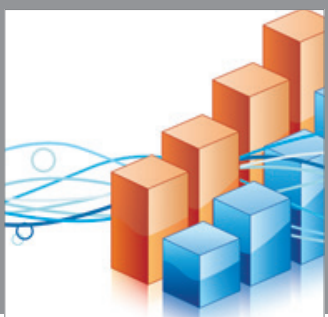

Advances in

Operations Research

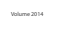

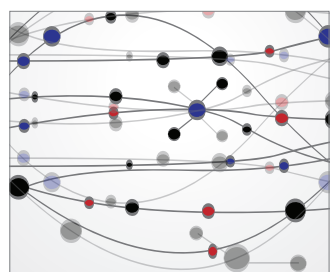

\section{The Scientific} World Journal
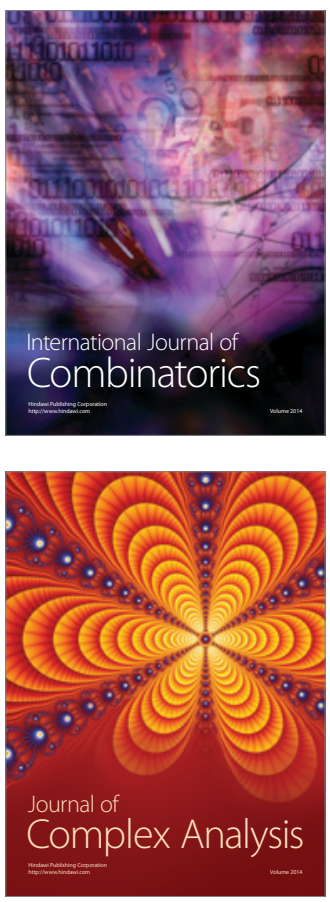

International Journal of

Mathematics and

Mathematical

Sciences
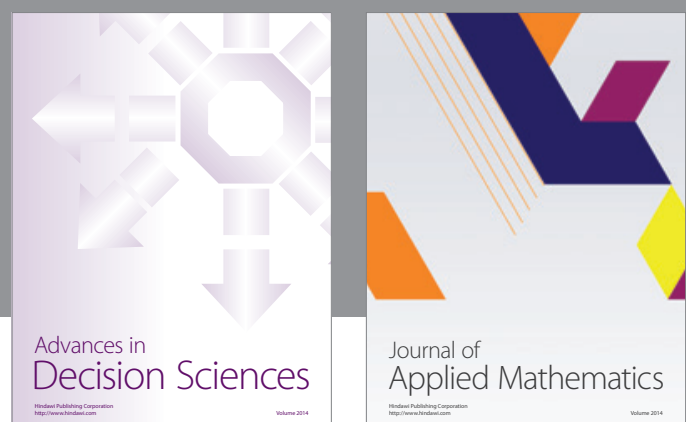

Journal of

Applied Mathematics
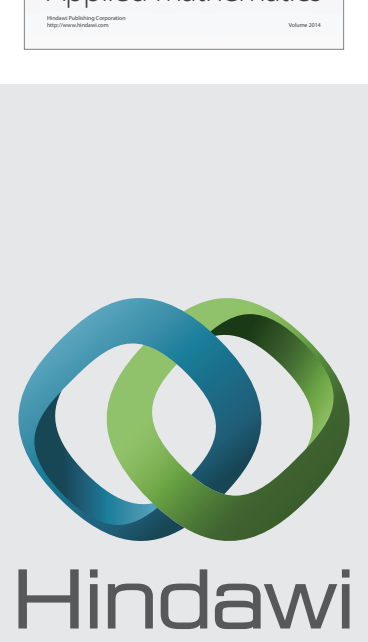

Submit your manuscripts at http://www.hindawi.com
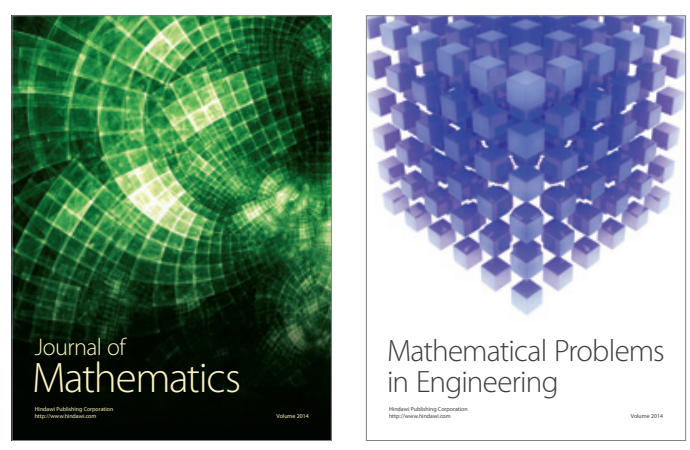

Mathematical Problems in Engineering
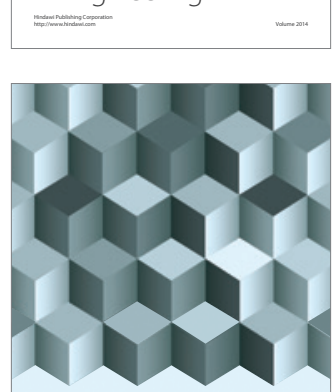

Journal of

Function Spaces
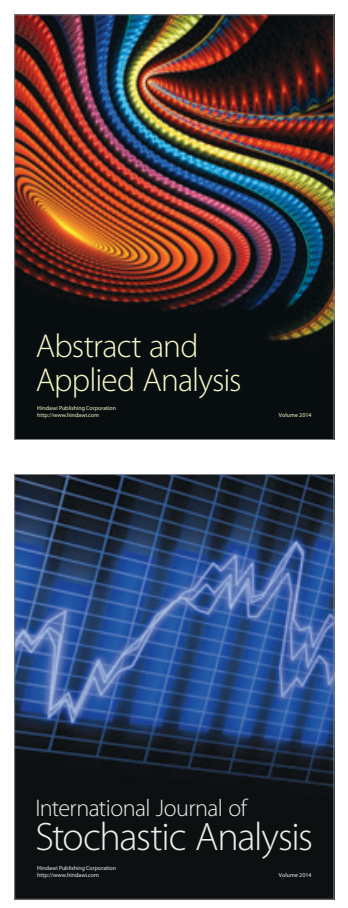

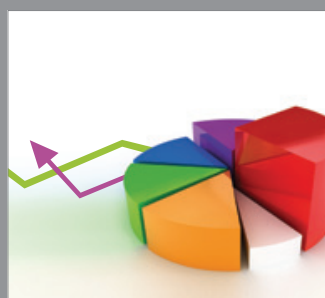

ournal of

Probability and Statistics

Promensencen
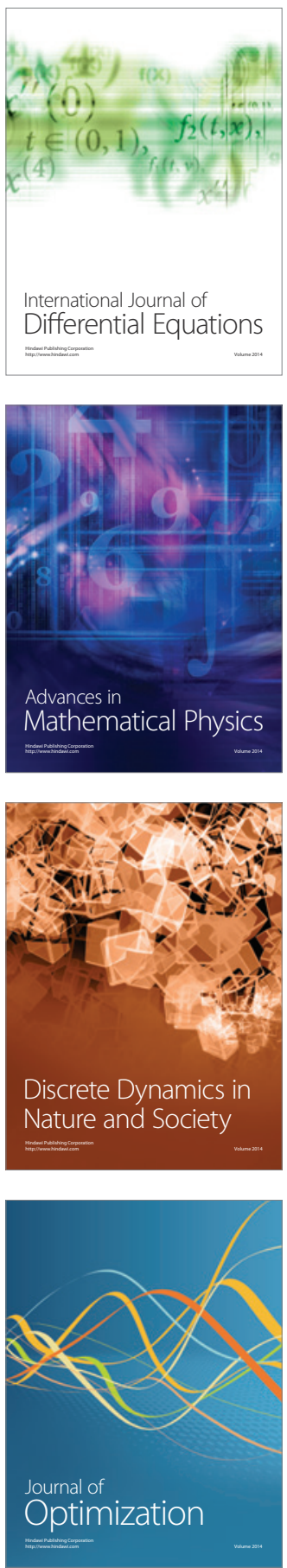